\title{
DATA ASSIMILATION USING SPATIAL PARTITION-BASED PARTICLE FILTERING FOR FREEWAY TRAFFIC SIMULATION
}

\author{
Yuan Long \\ Xiaolin $\mathrm{Hu}$ \\ Peisheng $\mathrm{Wu}$ \\ Georgia State University \\ Atlanta, GA 30303 USA \\ \{ylong4,xhu\}@gsu.edu, feng0905@gmail.com
}

\begin{abstract}
Assimilating real time sensor data into road traffic simulation is an important technique to achieve more accurate traffic simulation and prediction. A major challenge of traffic data assimilation is the high dimensional system states due to a large number of road segments whose states need to be estimated. To address this challenge, this paper adapts a spatial partition-based particle filtering method developed in earlier work and applies it to data assimilation for freeway traffic simulation. The spatial partition-based particle filtering method employs a divide and conquer strategy to reduce spatial complexity in state estimation. The developed method is applied to a discrete event freeway traffic simulation model based on Discrete Event System Specification (DEVS). Experiment results show the spatial partition-based particle filtering can effectively improve data assimilation results for freeway traffic simulations.
\end{abstract}

Keywords: Data assimilation, freeway traffic simulation, particle filtering, spatial partition.

\section{INTRODUCTION}

Traffic congestion is a serious problem in large cities and has major impacts on the economy and society. It is estimated that in each year, traffic congestion wasted 118 hours of time for an average driver (INRIX) (Burfeind 2018). Traffic simulation is an important tool for traffic management authorities in urban cities to help monitoring traffic patterns and predicting accidents or congestions. In the past decades, researchers have designed and proposed various traffic simulation models, such as Lighthill-Whitham-Richards (LWR) (Lighthill and Whitham 1955), Van Aerde (Van Aerde and Rakha 1995), MovSim (Treiber and Kesting 2010), and SUMO (Al-Akaidi 2002) to name a few. However, achieving accurate traffic simulations remains a challenging task due to model and data errors as well as the complexity of traffic behavior. To improve simulation accuracy, assimilating sensor data into traffic simulation is essential. Most existing works on data assimilating for traffic simulation are based on macro traffic simulation models (e.g., those defined by partial differential equations) and use Kalman filter (Harvey 1990) and its variants (Evensen 2003), which assume that the system behavior is linear. Traffic flow, however, is well known to have nonlinear non-Gaussian behavior. To deal with the non-linearity of traffic flow, there is increasing interest in carrying out data assimilation for traffic simulation based on Particle Filters (PFs) (Doucet and Gordon 2010).

PFs, also known as Sequential Monte Carlo methods (SMC) are state estimation methods that use a set of samples to approximate the posterior distribution of system states based on real-time observation data. For large spatial-temporal systems that cover large spatial areas of interest, the standard PFs may not work well 
due to the high dimensional system states (i.e., a large number of state variables) that need to be estimated (Snyder,Bickel and Anderson 2008; van Leeuwen 2009). One way of addressing this issue is to increase the number of particles, which can help increasing the diversity of samples and improving the state estimation accuracy. However, a very large number of particles will significantly increase the computation cost of data assimilation and pose challenges for real-time execution. Another way of addressing the high dimensionality issue is to design more advanced PF methods with new sampling/resampling techniques. In previous work (Long and Hu 2017), we developed a spatial partition-based particle filtering method for data assimilation in wildfire spread simulation. The developed method exploits the spatial locality property of system state and observation data and employs a divide-and-conquer strategy to reduce state dimension and data complexity. Experiment results showed that the method was able to improve the diversity of samples in state estimation and increase the simulation accuracy when using a limited number of particles.

The principle of spatial partition-based particle filtering can also be applied to data assimilation for road traffic simulations. Thus we intend to extend the method developed in previous work (Long and $\mathrm{Hu} 2017$ ), which was based on a 2D cellular space for wildfire spread simulation, to support data assimilation for road traffic simulations. Different from wildfire spread behavior that happens in a 2D Euclidean space, traffic flow is a phenomenon occurring over a traffic network composed of nodes (e.g., intersections) and links (road segments). Before considering general and complex traffic networks, this paper focuses on freeway traffic that has a relative simple network structure and develops and evaluates the spatial partition-based PF data assimilation method for freeway traffic simulation. A freeway traffic network is modeled by a sequence of road segments connected to one another (Boel and Mihaylova 2006; Mihaylova et al. 2007). The basic idea of spatial partition-based PF is to divide the road segments into multiple partitions, and correspondingly the overall system state is divided into multiple sub-states. During data assimilation, each sub-state's importance weight is computed based on the observation data belonging to its spatial partition. These importance weights of sub-states are then used by the resampling step to reconstruct new full states by selecting sub-states from different particles and compose them together. Comparing to the standard PF that treats system state as a whole during sampling, weight computation, and resampling, the spatial partition-based PF computes weights and reconstructs particles at the sub-states level. We note that the spatial partition-based PF method makes sense for road traffic data assimilation due to the spatial locality property of road traffic, which means the observation data and traffic states are spatially dependent and their immediate influences are constrained to their local spatial areas. For example, a traffic congestion in a specific road segment is reflected by the observation data in that area and has an immediate impact in the local area. We apply the spatial partition-based particle filtering to a discrete event freeway traffic simulation model based on DEVS (Zeigler et al. 2000) and present experiment results. We note that the developed data assimilation method does not depend on DEVS and can be applied to other freeway traffic simulation models.

The remainder of the paper is organized as follows. Section 2 describes the spatial partition-based PFs and how it is implemented for road traffic simulation. Section 3 presents the DEVS-based freeway traffic simulation model. Section 4 shows data assimilation results. Section 5 concludes this work.

\section{SPATIAL PARTITION-BASED PF FOR FREEWAY TRAFFIC SIMULATION}

\subsection{Overview of the Spatial Partition-Based PF Data Assimilation Framework}

The spatial partition-based particle filter proposed in (Long and $\mathrm{Hu}$ 2017) is designed to improve performance of data assimilation by employing a divide and conquer strategy for spatial temporal systems. The original method was developed based on a 2D cellular space for data assimilation in wildfire spread simulations. It takes advantage of the spatial locality of wildfire spread and observations by calculating the weights of sub-states using "local" observations and carrying out resampling based on the sub-states.

The developed spatial partition-based PF method consists of four steps. The first step is the same as the sampling step in a standard Bootstrap PF. In this step, each particle evolves to a new system state using the 
system transition model (the simulation model in our case). The second step is the state partitioning step. This step partitions the space into multiple sub-regions based on some criteria, e.g., physical boundaries and/or locations of sensors. Accordingly, the state represented by each particle is divided into multiple smaller sub-states based on the spatial partitions. The third step is the weight calculation step. This step differs significantly from the weight calculation in the Bootstrap PF. Instead of calculating the weight for a full system state, the spatial partition-based PF calculates the weight of each sub-state using the local observations related to the sub-states. The last step is the resampling step, which constructs new particles by resampling sub-states based on their weights and composing the sub-states into full system states. This step also differs from the standard Bootstrap PF that resamples particles at the whole-state level. Figure 1 shows the overall structure of the spatial partition-based PF data assimilation framework. More details and formal description of this framework can be found in (Long and $\mathrm{Hu} 2017$ ).

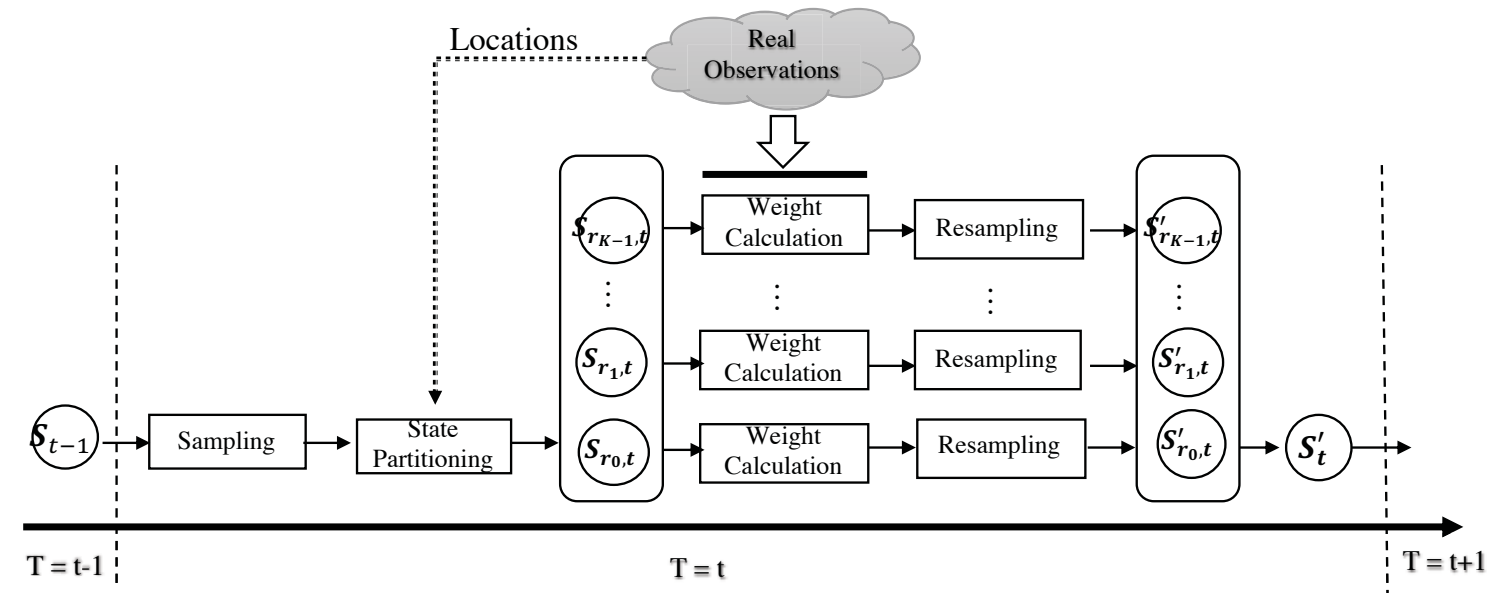

Figure 1: Spatial Partition-based PF data assimilation framework.

The original spatial partition-based PF was developed based on a 2D cellular space(Long and Hu 2017). It needs to be adapted in order to be applied to freeway traffic data assimilation. Following the structure of the original framework, below we describe each of the four steps (sampling, state partitioning, weight calculation, and resampling) of the spatial partition-based PF data assimilation for freeway traffic simulation.

\subsection{Sampling}

This step updates the state of each particle through the state transition model of the freeway traffic system. In our work, the state transition model is the traffic simulation model. Following previous work of freeway traffic simulation (Mihayloya et al. 2007), a freeway traffic system is modeled by a sequence of road segments. Let $M$ be the number of road segments of the freeway road. The state $s_{t}$ of the traffic system at time step $t$ can be represented as $\left\langle s_{t, 0}, \ldots, s_{t, M-1}\right\rangle$, which is a vector of states of all the road segments.

Note that the state transition model of the freeway traffic system requires a full system state in order to evolve to the next state. Therefore, at the beginning of the sampling step, we must ensure that a full system state has been obtained for each particle.

\subsection{State Partitioning}

After sampling the new system state, the state partitioning step breaks the full system state of each particle into multiple sub-states according to a specific spatial partition method (described below). For freeway road that consists of a list of linked road segments, we can divide the road space $r$ into $\mathrm{K}$ regions $\left\{r_{0}, \ldots, r_{\mathrm{K}-1}\right\}$ where $\mathrm{K} \leq \mathrm{M}$. Each region consists of a continuous sequence of road segments. Accordingly, we obtain a vector of sub-states $\left\langle s_{\mathrm{r}_{0}}, \ldots, s_{\mathrm{r}_{K-1}}\right\rangle$, each of which represents the state of its region. To give an example, 
consider a freeway road consisting of six road segments $\left\langle c_{0}, c_{1}, c_{2}, c_{3}, c_{4}, c_{5}\right\rangle$. The full system state is $<s_{0}, s_{1}, s_{2}, s_{3}, s_{4}, s_{5}>$, where $s_{\mathrm{j}}$ is the state of road segment $\mathrm{j}$. Assume we would like to partition the road into three regions: $r_{0}, r_{1}$ and $r_{2}$, where $r_{0}$ includes $c_{0}, r_{1}$ includes $c_{1}, c_{2}, c_{3}$, and $r_{2}$ includes $c_{4}, c_{5}$. Therefore, the full state $s$ is divided into three sub-states $s_{\mathrm{r}_{0}}, s_{\mathrm{r}_{1}}$ and $s_{\mathrm{r}_{2}}$ correspondingly. Each sub-state is composed of a vector of states corresponding to the road segments in the divided region. For instance, $s_{\mathrm{r}_{2}}$ is composed of state $s_{4}$ and $s_{5}$.

Our previous work (Long and $\mathrm{Hu}$ 2017) discussed several methods of partitioning a 2D cellular space for data assimilation in wildfire spread simulation. The main goal there was to achieve a high combination score by reducing the number of boundary sensors that have observation ranges covering multiple partitions in a 2D space. However, in this work we consider traffic speed sensors, which measure traffic speed within a narrow range nearby the sensors. Thus the boundary sensor issue (e.g., a speed sensor measures traffic speeds of more than one road segment) is not important. Nevertheless, freeway traffic has its own unique features. For example, traffic sensors typically are deployed sparsely and non-uniformly (in terms of distances from each other) along the freeway road. Due to these reasons, we propose two spatial partition methods for freeway traffic data assimilation as described below.

\section{1) Uniform partitioning}

In this straightforward spatial partitioning approach, the roadway is broken into multiple regions, each of which consists of an equal (or close to equal) number of road segments. By default, we assume the partition starts from the first road segment of the freeway. The drawback of this uniform partitioning is that some regions may have few or no sensor deployed there and thus there is zero or minimal observation data for those regions. This makes it ineffective to calculate the importance weights for the sub-states of those regions.

2) Balanced partitioning

Unlike the uniform partitioning, the balanced partitioning aims to balance the number of sensors over the divided regions. The goal is to find a balanced partition that makes every region have an equal number of sensors while keeping minimum road length variance. The balanced partition algorithm works as below:

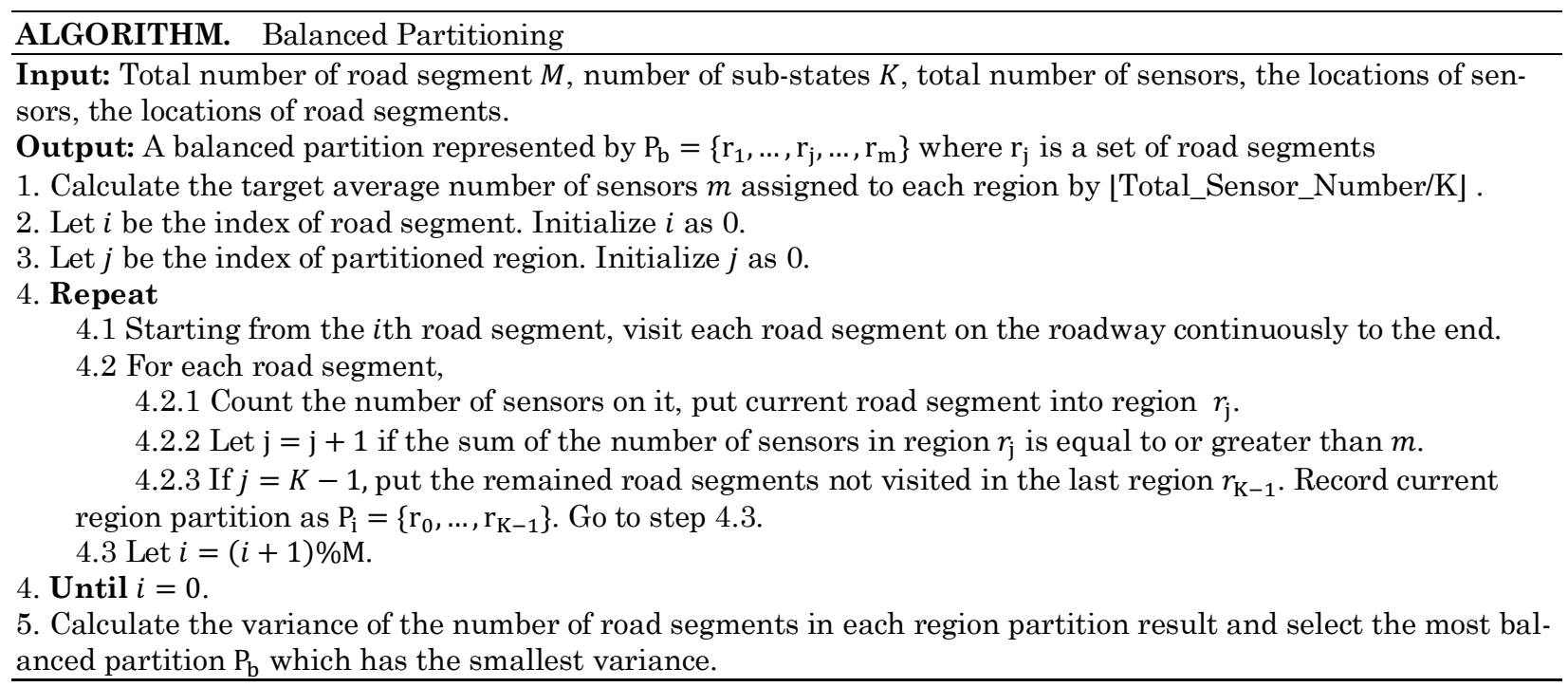

In above algorithm, given the number of sub-states $K$, we first calculate the target average number of sensors $m$ assigned to each sub-state by [Total_Sensor_Number $/ K\rfloor$. We represent the index of road segment using $i$ and let $i$ starts from 0 . Then we can find $K-1$ different region partitions by repeatedly visiting all road segments starting from the $i t h$ road segment. At the end of each iteration, the index of the 
starting road segment $i$ is moved to the next adjacent road segment. At each iteration, we obtain a partition of regions by counting the number of sensors so that each region has a number of sensors close to the target average number $m$. The iteration stops when the index reaches the initial road segment index. After all iterations, we calculate the variance of the number of road segments in every region partition and select the most balanced partition which has the smallest variance.

We note that the result of the balance partitioning depends on the specific locations of the sensors as well as the number of regions that need to be partitioned. The balance partitioning aims to achieve an equal (or close to equal) number of sensors to be assigned to each region. However, each region may not have the same number of road segments.

\subsection{Weight Calculation}

The step of weight calculation is to calculate the importance weights of particles based on observation data. Different from the Bootstrap PF, the spatial partition-based PF calculates a weight for each sub-state. Let $\omega_{s_{\mathrm{r}_{j}}}$ represent the weight assigned to sub-state $s_{t, r_{j}}$ in region $r_{j}$ for a particle. Therefore, the particle is associated with a weight set $\left\{\omega_{\mathrm{s}_{\mathrm{r}_{0}}}, \omega_{\mathrm{s}_{\mathrm{r}_{2}}}, \ldots, \omega_{\mathrm{s}_{\mathrm{r}_{\mathrm{j}}}}, \ldots, \omega_{\mathrm{s}_{\mathrm{r}_{\mathrm{K}-1}}}\right\}$ instead of a single weight. In general, if there are $C_{r_{j}}$ sensors located at region $\mathrm{r}_{\mathrm{j}}$, the weight of the sub-state for this region will be computed based on all these sensors. In this work, the sensors are traffic speed sensors and different speed sensors are assumed to be independent. Thus the weight of sub-state in region $r_{j}$ can be calculated by equation (1)

$$
\omega_{\mathrm{s}_{\mathrm{r}_{j}}}=\prod_{c=0}^{C_{r_{j}}} q\left(y_{r_{j}, c} \mid s_{r_{j}}\right)
$$

where $y_{r_{j}, c}$ is the reading from sensor c, $q\left(y_{r_{j}, c} \mid s_{r_{j}}\right)$ is the measurement model corresponding to the speed sensor describing the likelihood of observation $y_{r_{j}, c}$ given state $s_{r_{j}}$. In this paper, we represent the measurement model $q$ by a Normal distribution $N\left(y_{r_{j}, c}, \delta_{c}\right)$ where $\delta_{c}$ is the variance used for the $c t h$ observation.

\subsection{Resampling}

An important goal of the resampling step is to diverse the samples to alleviate the sample degeneracy problem of PF. To achieve this goal, we apply the traditional resampling method at the sub-state level (instead of at the whole state level) in which we generate a new set of samples through a probability proportional to the normalized weights.

First, we resample the particles for each sub-state. To do this, the sub-states from the same region $r_{j}$ but different particles are grouped as a sub-state set $\left\{s_{r_{j}}^{1}, \ldots, s_{r_{j}}^{N}\right\}$, where $\mathrm{N}$ is the total number of particles. For each sub-state set, the resampling is then carried out based on the normalized weights $\left\{\omega_{\mathrm{s}_{\mathrm{r}_{j}}}^{1}, \ldots, \omega_{\mathrm{s}_{\mathrm{r}_{j}}}^{N}\right\}$, in which weight $w_{\mathrm{s}_{\mathrm{j}}}^{i}$ is the importance weight associated to the corresponding sub-state $s_{r_{j}}^{i}$ (calculated in step 2.4). The resampled sub-state set corresponding to region $r_{j}$ is denoted as $\left\{s_{r_{j}}^{(1)}, \ldots, s_{r_{j}}^{(N)}\right\}$.

After that, we construct new particles by forming new full states based on the resampled sub-states. Specifically, a full state for a new particle $i$ is formed by selecting $s_{r_{j}}^{(i)}$ for all sub-states and then combining them into a new state $<s_{r_{0}}^{(i)}, \ldots, s_{r_{K-1}}^{(i)}>$. This step is important since the sampling step for the next iteration in the freeway traffic simulation requires a full system state as the input. 


\section{THE DISCRETE EVENT FREEWAY TRAFFIC SIMULATION MODEL}

We apply the spatial partition-based PF data assimilation framework to a DEVS-based discrete event freeway traffic simulation model (Hu and Wu 2018), named as DEVS-Traffic in this paper. This section gives an overview of this model while more details can be found in ( $\mathrm{Hu}$ and $\mathrm{Wu} 2018)$. Following previous work in the freeway traffic simulation literature (Boel and Mihaylova 2006; Mihaylova et al. 2007), DEVSTraffic models the freeway as a one-dimensional cellular space where the cells represent road segments as illustrated in Figure 2. Each cell keeps track of the positions and speeds of the vehicles within it using a FIFO queue. When a vehicle reaches the end of a segment it is added to the segment ahead (also named as the front segment) through a message passing. Figure 2 shows how the traffic is modeled when considering only four road segments in the roadway. The solid rectangles represent road segments. $\mathrm{N}$ represents the number of vehicles in each road segment. There are two pairs of input and output ports for each road segment. One pair is used to send and receive vehicle messages between segments. The other pair transmits the number of the vehicles in a segment to its rear segment (also named as the back segment).

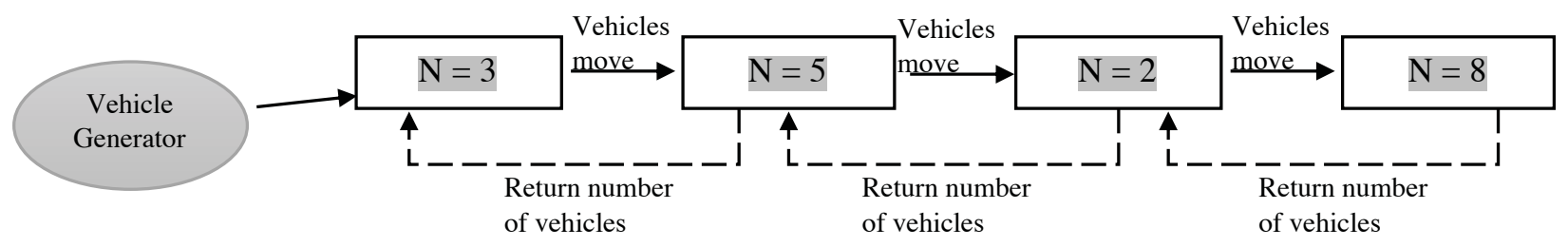

Figure 2: DEVS model of freeway traffic simulation.

All the vehicles in a segment travel at the same speed, which is determined by the number of vehicles in the segment as well as the number in the segment ahead. Roughly, the more congested the road and the road ahead the slower a driver must go. When the number of vehicles of a segment changes over time, it dynamically influences the vehicle speeds of its own segment and the back segment. Besides the vehicle number, the road condition of a segment (e.g., debris on the road) also has an impact on the vehicle speed. The road condition of the segment $i$ is modelled by a coefficient $\beta_{\mathrm{i}}$, whose value is between 0 and 1: 0 means the road is totally blocked (e.g., due to accidents) and 1 means the road is in normal condition Mathematical equations for calculating the vehicle speed of a road segment based on the road and front road segments' vehicles numbers as well as the road condition can be found in ( $\mathrm{Hu}$ and $\mathrm{Wu} 2018$ ).

Each road segment is modeled as a DEVS atomic model, which has a FIFO queue to store all the vehicles within it, including their specific positions on the road segment. The model calculates the speed and updates the positions of the vehicles within its FIFO queue based on external and internal events. A road traffic system is model as a DEVS coupled model that includes multiple road segment atomic models coupled together. To inject traffic into the road segments, vehicle generator models (as illustrated in Figure 2) can be added to generate traffic according to a distribution or pre-defined patterns. The vehicle generator is implemented as a DEVS atomic model and is similar to a typical generator model in DEVS. The DEVSTraffic model is developed based on previous work in the traffic simulation literature (Boel and Mihaylova 2006; Mihaylova et al. 2007). Simulation results of DEVS-Traffic shows that it was able to general similar behavior patterns as in (Boel and Mihaylova 2006; Mihaylova et al. 2007), including the forward and backward propagation of traffic congestions (Hu and $\mathrm{Wu} 2018$ ).

\section{EXPERIMENTS}

To evaluate the spatial partition-based PF for freeway traffic simulation, we apply it to the DEVS-Traffic simulation model and show experiment results. We use identical-twin experiment, which is commonly used in data assimilation research, to evaluate the performance of the proposed data assimilation framework. In the identical-twin experiment, we first run a simulation and consider the generated results as the ground truth data or "real results". The observations generated are recorded as the "real observations". After that, 
we inject errors or noises into the simulation model and estimate the simulation results by applying the data assimilation framework given the obtained real observations from the first run. We call the results generated in this simulation as the "filtered results". The smaller the difference between "real" and " filtered" results is, the better the state estimation from the data assimilation.

In this work, each road segment's state consists of two state variables: the FIFO vehicle queue that includes a list of vehicles, each of which has its own positions (spatial locations on the road segment), and the number of vehicles that it receives from the front segment ( $\mathrm{Hu}$ and $\mathrm{Wu} 2018)$. The overall system state consists of the system state from all the road segments. Based on this system state $t$, we can define the system transition model for the traffic simulation as follows in equation (2):

$$
s_{t}=\text { DEVS_Traffic }\left(s_{t-1}, \theta_{t}, \Delta t\right)+v_{t},
$$

where $\mathrm{s}_{\mathrm{t}-1}$ and $\mathrm{s}_{\mathrm{t}}$ are the system state at data assimilation step $\mathrm{t}-1$ and step $\mathrm{t}, \Delta t$ is the time duration between the data assimilation steps, $\theta_{t}$ is a vector of model input (road condition and vehicle generator) and $\mathrm{v}_{\mathrm{t}}$ is the system noise.

\subsection{Experiment Settings}

To carry out experiments, we configured the freeway traffic system as a circular roadway (e.g., a ring freeway encircling a city) constructed by 60 road segment atomic models (see Figure 5). The road segment has index starting from 0 to 59. Each road segment is 100 meters long and can accommodate at most 34 cars. To initialize the state of the road system before the data assimilation, a traffic generator is used to inject traffic into road segment 0 . The traffic generator produced vehicles sequentially for 750 seconds, and then the data assimilation starts. It keeps generating the vehicles until another 250 seconds passed. There are 15 data assimilation (DA) steps in a single run. The length of a data assimilation step is 20 seconds.

Table 1: Road Condition Coefficients in Experiment Configuration.

\begin{tabular}{lll}
\hline Time Step & Real System & Simulations with errors \\
\hline $\mathrm{T}=2$ & Set $\beta_{48}=0.1$ & 1 on all road segments \\
$\mathrm{T}=4$ & Set $\beta_{12}=0.1, \beta_{24}=0.15$ & Same as above \\
$\mathrm{T}=6$ & Set $\beta_{48}=1, \beta_{36}=0.1$ & Same as above \\
$\mathrm{T}=8$ & Set $\beta_{12}=1, \beta_{24}=1$ & Same as above \\
$\mathrm{T}=13$ & Set $\beta_{36}=1$ & Same as above \\
\hline
\end{tabular}

Table 1 shows the different settings between the "real" and "filtered" simulations described previously. In the simulation for the "filtered" results, road condition coefficient $\beta$ of each segment was set as 1 . During the "real" simulation, the road condition coefficients $\beta$ varied at some road segments due to the accidents or other events. For example, the coefficient of road segment 48 started at 0.1 and changed to 1 at DA step 7. A small road coefficient can lead to the heavy traffic on the roadway. So, there should be multiple congestions in the "real" traffic at some certain time steps. Figure 3 shows the "real" traffic patterns at different DA assimilation steps (the black line). As can be seen, at step $\mathrm{T}=6$, there are multiple congestions locations along the road; at time $\mathrm{T}=11$, the congestions location reduces to one; and at $\mathrm{T}=15$, the traffic smooths out. The goal of data assimilation is to estimate the traffic state for all road segments based on observation data from limited sensors deployed on the roadways. The estimated system states are then used to run simulations to achieve more accurate traffic predictions.

To study the impact of spatial partition-based PFs, we conducted two sets of experiments by varying the number of partitions (i.e., sub-states), and partitioning methods separately. In the first set of experiments, the sensors were distributed regularly on the roadway. Specifically, sensors are deployed regularly with one 
sensor in every two road segments. In the second set of experiments, we considered the case when sensors were distributed unevenly on the roadway and observed the traffic state using 30 sensors. These 30 sensors were generated by placing 15 sensors uniformly on the roadway first and then another 15 sensors uniformly within the first half of the roadway. Figure 5 shows the locations of the deployed sensors (the gray cells have sensors and the white cells do not). Every experiment run 5 times, and we report the averaged results from the 5 runs.
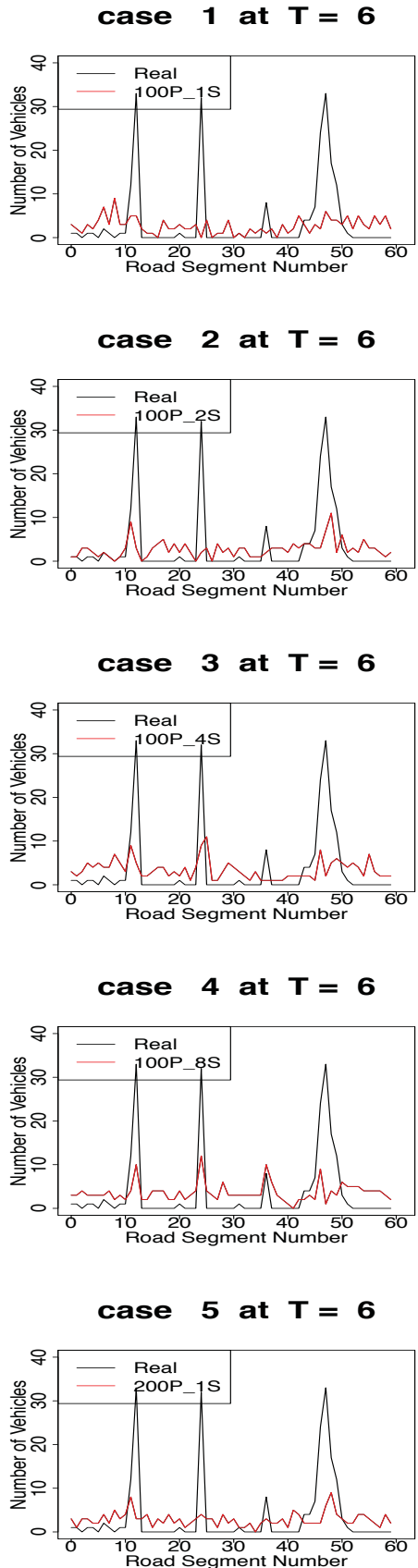

case 1 at $T=11$

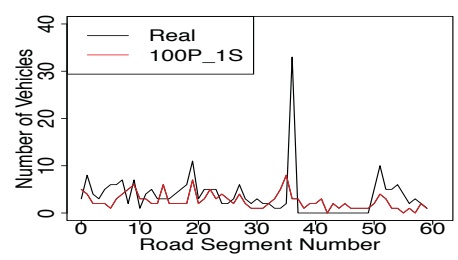

case 2 at $T=11$

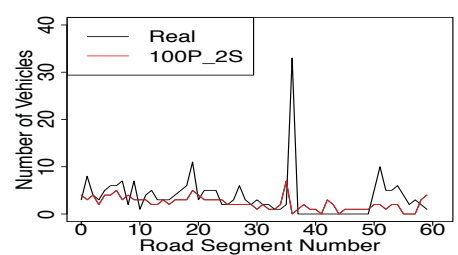

case 3 at $T=11$

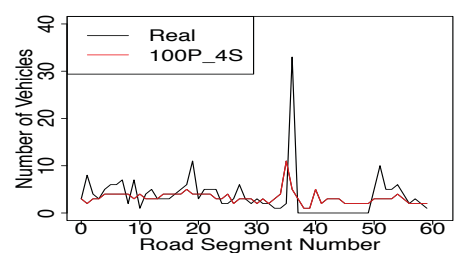

case 4 at $T=11$

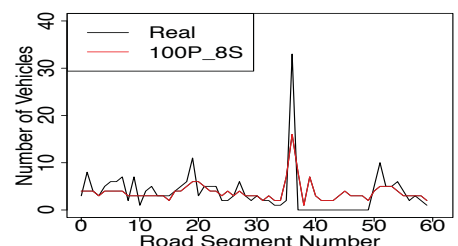

case 5 at $T=11$

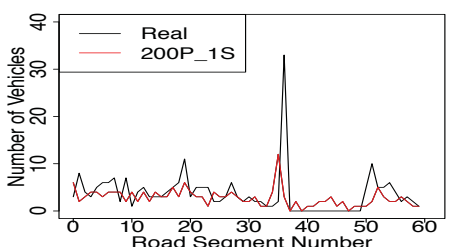

case 1 at $T=15$

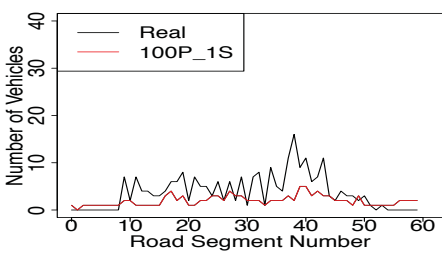

case 2 at $T=15$

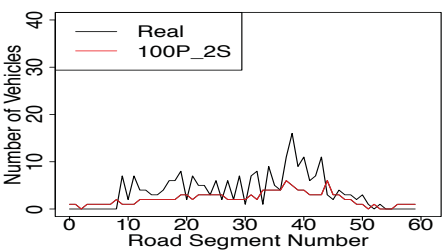

case 3 at $T=15$

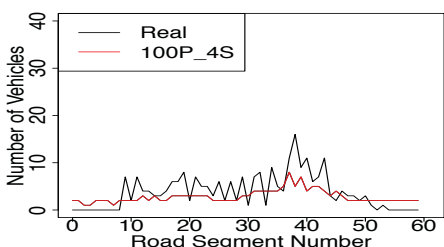

case 4 at $T=15$

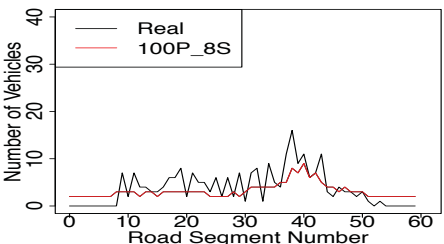

case 5 at $T=15$

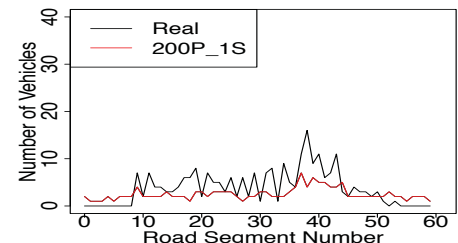

Figure 3. Comparison of traffic system state for the five cases. In each chart, P represents the particles, $\mathrm{S}$ represents sub-states. 
We use the measurement root-mean-square errors (RMSEs) to represent the accuracy of the filtered result. The RMSE at time step $t$ was calculated by the equation below

$$
R M S E_{t}=\sqrt{\frac{\sum_{i=1}^{N}\left(s_{t}^{i}-\text { Real_s }_{t}^{i}\right)^{2}}{N}},
$$

where $i$ is the $i t h$ road segment and $N$ is the total number of road segments, $s_{t}^{i}$ and Real_s ${ }_{t}^{i}$ represents the estimated vehicle number from the data assimilation and that of the real system.

\subsection{Experiment Results and Analysis}

1) Number of Partitions

The roadway was divided into equal 1,2, 4 and 8 partitions separately. We tested all the four partitions respectively by using 100 particles. We also experimented the case when using 200 particles and one full state (i.e. the state is not divided). In total there are five cases compared. Case 1 is 100 particles, one state, denoted as 100P_1S; Case 2 is 100 particles, two sub-states, denoted as 100P_2S; Case 3 is 100 particles, four sub-states, denoted as 100P_4S; Case 4 is 100 particles, eight sub-states, denoted as 100P_8S; Case 5 is 200 particles, one state, denoted as 200P_1S.

The simulation results at DA step 6, 11, and 15 for all five cases are displayed in Figure 3 separately. Each sub-chart compares the number of vehicles on the road segments between real simulation and filtered simulation. Black lines show the results from the "real" simulation. The red lines denote the results from the filtered simulation. From the "real" simulation, it is not hard to obtain the "real" road traffic pattern. Initially, there were three main congestions over the roadway. Eventually, these congestions diminished when road condition coefficient changed back to 1 and no more new cars injected into the traffic.

We evaluate the impact of the number of sub-states on the prediction performance by checking the subcharts column by column. It can be seen that more congestions were detected when the number of substates was increased. When the simulation space is large, it is hard to accurately predict the locations of multiple congestions using a limited number of particles with insufficient observation. But if the simulation space is smaller, the prediction on traffic congestion at a smaller space would be more accurate since the located region is covered with sufficient observation.

RMSE

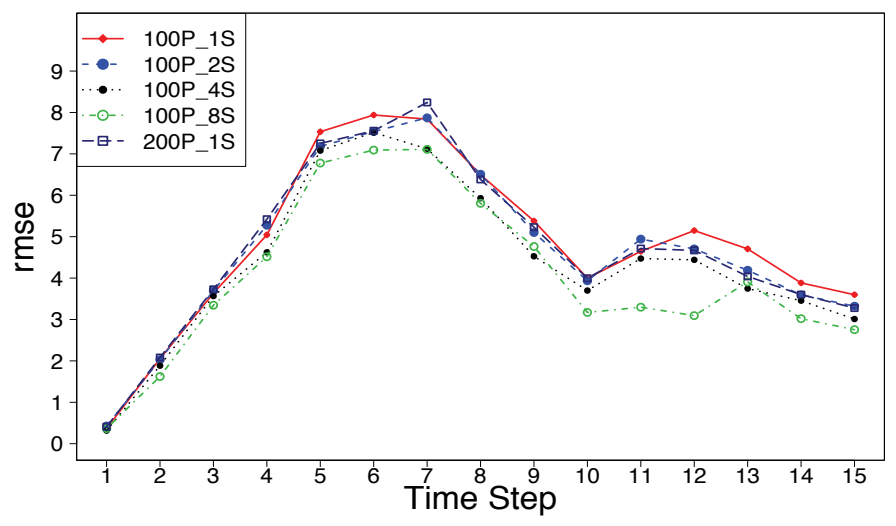

Figure 4: Comparison of RMSEs for the cases when varying the number of partitions.

Figure 4 shows the numeric results of RMSEs. Initially, the RMSEs increased when traffic became more congested. This trend did not stop until the DA step 7 when the road condition was the worst in the "real" system. Before DA step 7, because of the incorrect road coefficients, the simulation model used in the filtered simulation differed a lot from the "real" system and therein brought errors to the filtered simulation. 
However, the RMSEs decreased with the diminishing of traffic congestion and inject of correct settings of road coefficients. By comparing the RMSEs of different cases for a given step, we can also see that as the number of sub-states increases, the data assimilation results improves in most steps. In particular, when the number of sub-states equals to 8 (i.e., the largest number of sub-states considered in this experiment) the RMSE is the minimum in all the steps.

\section{2) Partitioning methods}

We tried uniform and balance partitioning methods and compared their results accordingly. We note that the uniform partitioning was applied in all the cases of the first set of experiments. In this set of experiments, we divided the state into four sub-states. Figure 5 shows how the sensors were deployed on the roadway and how the state was partitioned by applying different partitioning method. Although uniform partitioning method balanced the number of road segments on each partition, it failed to balance the number of sensors on each partition. For example, sub-state \#4 was observed by only two sensors in the results of uniform partitioning as shown in Figure 5(a). Even though the number of road segments differ in the result of balance partitioning, each sub-state were still observed by an similar number of sensors shown in Figure5(b).

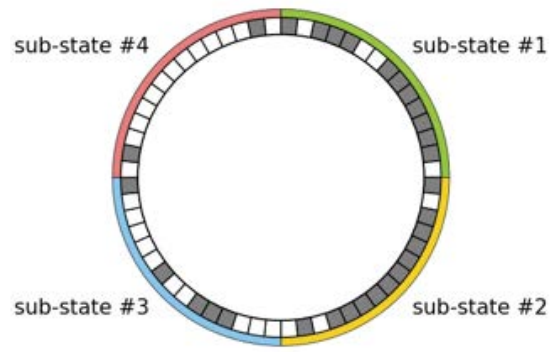

(a) Uniform Partitioning.

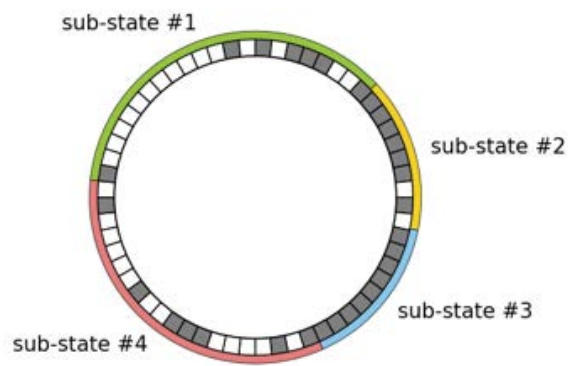

(b) Balance Partitioning.

Figure 5: Two Partitioning results generated by uniform partitioning and balance partitioning respectively. Each small grid on the circle represents a single road segment. The road segments observed by sensors are filled in gray and are divided into four groups in different colors.

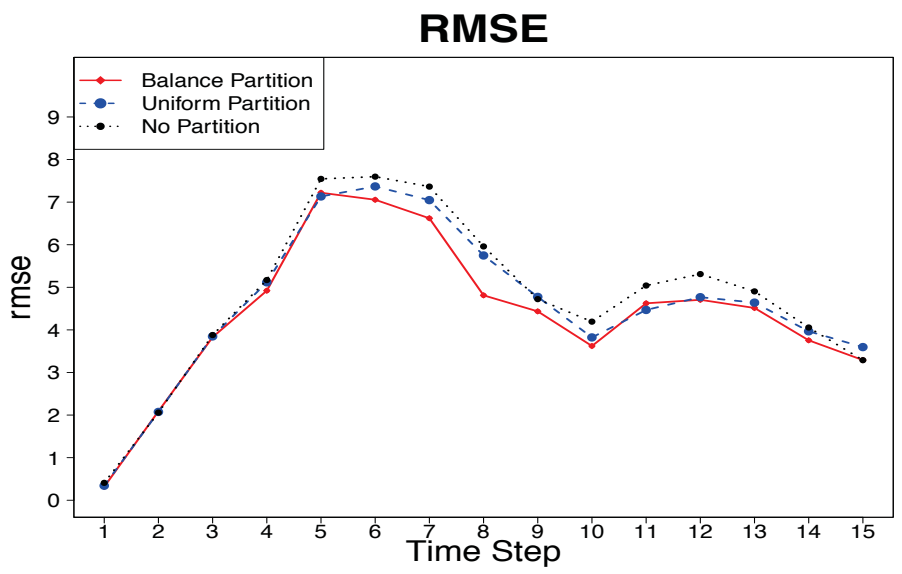

Figure 6: Comparison of RMSEs for the cases when road way was partitioned by different partitioning methods.

Figure 6 compares RSMEs for these two different partitions and the case the state was not divided. One hundred particles were used in this experiment. We can see that the RSME for the balance partitioning outperformed the other two partitions. This is because the balance partition guarantees that there is an equal number of sensors in the sub-region where the sub-state locates at. However, as seen in Figure 5 (a), some sub-regions have 3 or 4 sensors while others have around 10 sensors. In the uniform partition, when a sub- 
region has no sensors or enough sensors covered, the sub-state falling into this area cannot be observed correctly, and the poor prediction happened at the last time step when the traffic congestion appeared at this region. So, when partitioning a space, it is critical to take the sensors' observation coverage as important factors.

\section{CONCLUSION}

In this paper, we adapted and extended the spatial partition-based PF developed in an earlier work and applied it to data assimilation for freeway traffic simulation. Experiment results show that the spatial partition-based PF can effectively improve the data assimilation results. Future work include further development of the framework for complex and general traffic network structures, and developing more advanced spatial partition approaches for general traffic network structures.

\section{ACKNOWLEDGMENTS}

This work is supported in part by the US Department of Agriculture (USDA) National Institute of Food and Agriculture (NIFA) under grant number 2019-67021-29011.

\section{REFERENCES}

Al-Akaidi, A. . 2002. "SUMO (Simulation of Urban MObility) - an open-source traffic simulation". In Proceedings of 4th Middle East Symposium on Simulation and Modelling, 2002-09, Sharjah (United Arab Emirates), 183-187.

Boel B., L. Mihaylova. 2006. "A compositional stochastic model for real-time freeway traffic simulation". Transportation Research B, Vol. 40 No. 4, 319-334.

Burfeind M. . "Los Angeles Tops INRIX Global Congestion Ranking". Inrix.com. http://inrix.com/pressreleases/scorecard-2017/ (accessed February 5, 2018)

Doucet, A.N. Freitas, and N. Gordon . op. 2010. "An Introduction to Sequential Monte Carlo Methods". in Sequential Monte Carlo methods in practice, A. Doucet, N. de Freitas, N. J. Gordon and A. F. M. Smith (eds.), New York: Springer, 3-14.

Evensen, G. . 2003. "The Ensemble Kalman Filter: theoretical formulation and practical implementation". Ocean Dynamics. 53(4): 343-367.

Harvey, A. C. . 1990. Forecasting, structural time series models and the Kalman filter, Cambridge: Cambridge University Press.

$\mathrm{Hu}, \mathrm{X}$. , and P. Wu . 2018. "A Data Assimilation Framework for Discrete Event Simulations". ACM transactions on modeling and computer simulation. Accepted, to appear.

Lighthill, M. J., and G. Whitham . 1955. "On Kinematic Waves. II. A Theory of Traffic Flow on Long Crowded Roads". Proceedings of the Royal Society A: Mathematical, Physical and Engineering Sciences. 229(1178): 317-345.

Long, Y., and X. Hu . 2017. "Spatial Partition-Based Particle Filtering for Data Assimilation in Wildfire Spread Simulation". ACM Transactions on Spatial Algorithms and Systems. 3(2): 1-33.

Mihaylova, L., R. Boel, and A. Hegyi . 2007. "Freeway traffic estimation within particle filtering framework". Automatica. 43(2): 290-300.

Snyder, C.T. Bengtsson, P. Bickel, and J. Anderson . 2008. "Obstacles to High-Dimensional Particle Filtering". Monthly Weather Review. 136(12): 4629-4640.

Treiber, M., and A. Kesting . 2010. "An Open-Source Microscopic Traffic Simulator". IEEE Intelligent Transportation Systems Magazine. 2(3): 6-13. 
Van Aerde, M., and H. Rakha . 1995. "Multivariate calibration of single regime speed-flow-density relationships [road traffic management]". In Proceedings of Pacific Rim TransTech Conference. 1995 Vehicle Navigation and Information Systems Conference Proceedings. 6th International VNIS. A Ride into the Future, 30 July-2 Aug. 1995, Seattle, WA, USA, 334-341.

Van Leeuwen, P. J. . 2009. "Particle Filtering in Geophysical Systems". Monthly Weather Review. 137(12): 4089-4114.

Zeigler, B. P., Praehofer, H., and Kim, T. G. . 2000. Theory of modeling and simulation: Integrating discrete event and continuous complex dynamic systems / Bernard P. Zeigler, Herbert Praehofer, Tag Gon Kim, San Diego, Calif., London: Academic.

\section{AUTHOR BIOGRAPHIES}

YUAN LONG is affiliated with Georgia State University. Her research interests include machine learning, big data analysis and high performance computing. Her email address is ylong4@gsu.edu.

PEISHENG WU received his Ph.D. Degree from Georgia State University, and currently is a software engineer at CareerBuilder. His research interests include modeling and simulation and software engineering. His email address is feng0905@gmail.com.

XIAOLIN HU is an Associate Professor in the Department of Computer Science at Georgia State University, Atlanta, Georgia. He received his Ph.D. degree from the University of Arizona in 2004. His research interests include modeling and simulation theory and application, agent and multi-agent systems, and complex systems science. His email address is xhu@cs.gsu.edu and his web page is https://grid.cs.gsu.edu/xhu/. 